\title{
Targeting the Seven Cancer Hallmarks by Modulation of Oxidative Stress-induced Inflammation and Immune Activation: A Radical Therapeutic Approach
}

\author{
Mburu Samuel \\ School of Health Sciences, Kirinyaga University, Kenya
}

Copyright $(2017$ by authors, all rights reserved. Authors agree that this article remains permanently open access under the terms of the Creative Commons Attribution License 4.0 International License

\begin{abstract}
Cancer killed approximately 8.8 million people in 2015 globally. Furthermore, more than 27,000 Kenyans die annually from cancers, making it number three killers after infectious and cardiovascular diseases. The current therapeutic strategies are limited in their approach, therefore not effective enough to achieve complete remission. A radical multifactorial approach targeting early events in carcinogenesis is required. The purpose of this descriptive study was to review existing studies for knowledge, research gaps in the role of oxidative stress, inflammation, immune activation in carcinogenesis and cancer hallmarks, to stimulate new research ideas which can accelerate future therapeutic target discoveries. PubMed, ScienceDirect and Google scholar databases were searched using the keywords: cancer, oxidative stress, inflammation, immune activation, carcinogenesis and cancer hallmarks. Although widely recognized, little research on oxidative stress, inflammation, immune activation, as cancer therapeutic targets has been done. In addition, studies relating oxidative stress, inflammation, immune activation with cancer hallmarks, especially replicative immortality, immune evasion, and evading growth suppression are inadequate. To highlight this, out of a total of 8,680,095 hits, only 139,694 hits related to oxidative stress, inflammation, immune activation as therapeutic targets making this area a fertile ground for future research. Similarly, out of 271, 194 hits, only 4,595 were relating oxidative stress, inflammation and immune activation with replicative immortality as a cancer hallmark. Subsequently, after pearling, 129 articles that were directly relevant to the study were selected. After critical appraisal, identified studies were analyzed, results compared and presented in form of summary tables. Despite enough documented evidence of the essential role oxidative stress, inflammation, immune activation, plays in carcinogenesis, specific role in induction of cancer hallmarks, whether causal or consequence is not clear. An understanding of the early changes that marks initiation, maintenance and progression of cancer will accelerate
\end{abstract}

development of future novel therapeutic targets and prevention strategies. This will have a direct impact on prevention, early diagnosis, management and treatment of cancers in Africa, thereby helping in attainment of United Nations sustainable development goal (SDG) number three.

Keywords Cancer, Oxidative Stress, Inflammation, Immune Activation, Cancer Hallmarks, Carcinogenesis

\section{Introduction}

Cancer killed approximately 8.8 million people globally in $2015,70 \%$ of which were in low and middle income countries such as Kenya, thus making it a major cause of mortality [1]. Notably, about 40,000 (or about 80 deaths daily) new cases of cancer and 27,000 deaths occurs annually in Kenya, accounting for $7 \%$ of overall national mortality [2]. Consequently, cancer has become the third leading cause of death after infectious and cardiovascular diseases (CVDs) [3]. Globally, lung, liver, colorectal, stomach and breast cancers are the most common causes of cancer deaths [1]. However, in Kenya, esophageal, cervical cancers are the leading cause of cancer mortality in men and women. Alarmingly, stomach, liver, colon and rectal cancers are on the increase in Kenya [4]. In Children, leukemia and lymphomas are the most prevalent cancers globally [5]. Significantly, Human papilloma virus (HPV), a virus that is sexually transmitted accounts for $22 \%$ and $12 \%$ of all cancers in women and men respectively [6].

Of major concern, is the low level of public awareness, lack of knowledge and misinformation on cancer [7, 8]. For that reason, majority of cancers are diagnosed at advanced stages contributing to the high mortality rates from the disease. To highlight this, $80 \%$ of all cancers in Kenya are diagnosed at advanced stages [9, 10].

Cancer does not refer to a single ailment, but several 
different diseases. There are more than 200 different diseases classified as cancers or types of cancer [11]. American joint Committee on Cancers, (2007), has broadly classified cancers based on either the site of location of the malignant cells, histology or cell analysis, the extent of the disease (staging), organ where it first developed or histologically depending on the tissue it originates from [12]. Accordingly, carcinoma refers to cancer originating from the skin; leukemia originates in the blood, lymphomas from the cells of the immune system, myeloma from the immune system and sarcomas from the bone [13]. The stage of cancer at the time of diagnosis is critical in defining treatment, evaluating success of therapies and chances of recovery. The extent of the disease otherwise referred to as cancer staging can be done using various methods. These include; the summary stage system, surgical resection margin evaluation (R classification), the "extent of disease" system and the most widely used tumour, node, metastasis (TNM) system $[10,12]$. The TNM uses tumour size, degree of regional spread or node involvement and distant metastasis to classify cancers [12]. In addition to the stage, trait or the type of cells or tissues affected, social and psychological wellbeing of the patient influences greatly recovery in cancer $[14,15]$.

Essentially, cancer is multi-aetiology in nature that includes; genetic, lifestyle, biochemical, biological, environmental, chemical exposure factors [16-21]. However, the fraction of cancers attributable to lifestyle and environmental factors is $43 \%$, indicating that the origin of $57 \%$ of all cancers is still not clear [22]. Hence, more research is required to define aetiology of these cancers. This understanding will help $\mathrm{n}$ accelerating therapeutic target discovery, development of preventive and early diagnosis strategies. Common risk factors for cancer include; diet, lifestyle (sedentary, obesity, stress), reproductive, sexual behavior (for ovarian \& cervical cancers), occupational exposure to chemicals, pesticides, herbicides, heavy metals, some medical therapies such as ionizing radiation used in x-rays, alkylating compounds, geophysical factors, infectious agents or biological agents (such as bacteria, viruses, aflatoxin), chronic inflammatory diseases including diabetes mellitus, human immunodeficiency syndrome/acquired immunodeficiency syndrome (HIV/AIDS), tobacco smoking, chewing, exposure to secondary tobacco smoke, alcohol consumption and automobile fuel exposure $[16,17,21,23]$.

Several chemical factors have been identified as carcinogens such as; primary, secondary cigarette smoke, pesticide, herbicide e.g. dichloro-diphenyl-trichloroethane (DDT), lindane, oxyfluorfen, methoxychlor (which replaced DDT), chlorothalonils and atrazine. Similarly, other chemical carcinogens include; vinclozolin, 2-amino-1-methyl-6-phenylimidazo (4,5-b) pyridine (PhiP), a heterocyclen amine from meat preparation, synthesis of polycarbonates, epoxy resins, packaging thermal papers, plastic papers, bottles, water pipes, electronic equipment as well as toys, which is linked to breast, colon and prostate cancers [24-26]. Likewise, 4-Nonylphenol (found in contaminated food, water from liquid detergents), cosmetics, paints and pesticides are also common carcinogens. Alarmingly, Phthalates, a common compound used to soften plastics, make infusion sets for blood transfusion and from plastic packaging of foods, drinks and common in developing world, is carcinogenic if inhaled or absorbed through skin $[22,26]$. Of concern, acetaldehyde, a by-product of organic compounds such as alcohol, reported to activate nuclear factor of Kappa B (NF-kB), activator protein-1 (AP-1) and p38 signaling pathways has been associated with liver cancer [27].

In addition, heavy metals such as hexavalent chromium from industries, fossil fuels, cadmium are associated with testicular, prostate, adrenal and lung cancers [28, 29]. Other common carcinogens include; metals such as nickel, arsenics, (from inorganic farming of rice, vegetables and fruits), mercury, lead in detergents, cosmetics and asbestos, which is associated with lung cancer. Importantly, biological agents such as bacteria (e.g. H, pyroli), viruses (HIV, Epstein Barr Virus [EBV], Cytomegalovirus [CMV], Human papilloma virus [HPV], Hepatitis $B$ and $C$ ) and aflatoxins are also common carcinogens.

\subsection{Oxidative Stress, Inflammation, Immune Activation and Carcinogenesis; Partners in Crime}

Aetiology whether by biological agents, (bacteria $[H$. pylori], viruses [HIV, EBV, HPV, Hepatitis $B \& C]$ ), environmental, chemical carcinogens, chronic inflammatory conditions notwithstanding, a common characteristic of cancers is increased oxidative stress, inflammation, immune activation especially in the tumour microenvironment and bone marrow [21,30,31,32]. Interestingly, majority of cancers, if not all, starts in an environment of oxidative stress, inflammation and immune activation [30].

The influence of free radicals on proto-oncogenes, activation of oncogenes, initiation, promotion and evolution towards malignancy has been known from as early as 1990 [33]. Significantly, oxidative stress has been recognized as the "master key" through which tumour microenvironment interacts with almost all cancer hallmarks [23,34]. In addition, oxidative stress has been recognized as powerful initiator of the consistent NF-kB activation strongly associated with chemical, tobacco, alcohol, high-fat diet, environmental pollutants tumorigenesis, viruses, bacteria-associated cancers, ultraviolet light, ionization radiation and obesity-associated cancers [35,36,37].

Similarly, an altered tissue microenvironment is important during early stages of carcinogenesis when focal proliferative lesions (nodules/Adenomas, polyps and papilloma) appear [38]. Accordingly, increased oxidative stress in the tumour microenvironment is thought to cause oxidative deoxynucleic acid (DNA) damage in the stroma stem cells, initiation of focal proliferative lesions leading to 
activation of an inflammatory response [39]. Thereafter, a unique tumour environment emerges, characterized by defective oxygen, nutrients that lead to hypoxia and competition for the scarce resources [30]. This causes a vicious cycle of oxidative stress, inflammation, immune activation, ultimately leading to metabolic reprogramming, more genetic instability, DNA damage, induction of "driver mutations" in proto-oncogenes (Ras and Myc) and tumour suppressor genes (p53 and $\mathrm{Rb}$ ) [30,40].

Alternatively, chemical exposure from pesticides or herbicides may interact with the hypothalamic-pituitary-adrenal axis, leading to increased output of cortisol by the adrenals. This activates tumour-associated inflammatory (TIA) response mechanisms [41]. Subsequently, the cortisol produced impacts on several cytokines such as macrophage migration inhibiting factor (MIF), a potent pro-inflammatory cytokine, which links the HPA axis to the inflammatory process [42, 43]. Interestingly, this phenomenon explains the association between stressful lifestyle, poverty, exposure to environmental chemicals and the unexplained increased risk of cancers in poor populations [21].

Importantly, persistent NF-kB signaling ultimately prevents resolution of TIA, resulting in a localized milieu of chronic cytokine activation. This is believed to induce the cancer hallmarks such as; resistance to cell death, evading growth suppression or replicative immortality probably as an adaptation to local microenvironment oxidative stress. Furthermore, it promotes angiogenesis, evasion, metastasis as the tumour cells escape from the toxic tumour microenvironment, increased competition for oxygen and nutrients $[30,44]$.

Inflammation, which is an immediate defense response to foreign substances, tissue injury, or altered self-molecules, plays a causal role in viral, tobacco and asbestos-related carcinogenesis [45]. In addition to increasing the risk of developing cancer, about $20 \%$ of cancer deaths are caused by the chronic inflammation [46]. Notably, tumour-associated chronic inflammation, though commonly associated with infections, can also be triggered by exposure to irradiation, environmental and chemical carcinogens [47]. This is characterized by expression of tumour antigens, cytokines production (IL-8, TGF- $\beta$, TNF- $\alpha$, IL-I and 6), induction of immunosuppressive FOXP3 + T-regulatory cells (T-regs) [48], down regulation of major histocompatibility complex I (MHC I) molecules on cytotoxic T-cells (CD8 T-cells) [49,50] and dysfunctional CD8 T-cells [51]. All these events are believed to be driven by oxidative stress, inflammation and immune activation $[52,53]$.

Tumour microenvironment is composed of blood vessels, extracellular matrix and stroma or mesenchymal stem cells such as fibroblasts, endothelial cells, fat cells, macrophages, antigen presenting cells (APCs) such as dendritic cells and reticulum cells [54]. All these cells are labelled the "NOX family" as they have a nicotinamide adenine dinucleotide phosphate (NADPH) oxidase, in addition to xanthine oxidase and nitric oxidase systems $[55,56]$. These cells therefore, are major source of reactive oxygen species (ROS) and oxidative stress. Too, they are capable of a respiratory burst, which is a sequential reduction of oxygen by an NADPH oxidase system, to kill invading microorganisms, generating ROS. Essentially, xanthine oxidase system is regulated by lipopolysaccharide (LPS), pro-inflammatory cytokines and hypoxia [55].

Activation of the tumour microenvironment stroma/mesenchymal stem cells by cancer causing infectious bacteria, viruses and chemical carcinogens leads to oxidative stress, genomic instability, inflammation and immune activation, which causes the initial focal proliferative lesions [30, 38, 47, 57]. These lesions, which are as a result of damage to macromolecules (DNA, lipids, proteins etc.) by ROS, subsequently leads to an inflammatory response $[21,58,59,60]$. The initial reaction to the lesions, early tumour cells is recruitment, activation of multipotent stroma/mesenchymal stem cells, fibroblasts, endothelial cell precursors, APCs, white blood cells such as CD8-T lymphocytes and T-regs [61].

Hanahan et al. (2011) [61], indicated that the recruited normal cells play an active role in tumourigenesis as major source of ROS and oxidative stress $[55,56]$. These cells generate pro-inflammatory cytokines (TNF, IL-I \& IL-6), growth factors such as vascular endothelial growth factor (VEGF) which stimulates angiogenesis, cytokines (e.g. CXCL-10) and chemokines [62]. Consequently, a vicious cycle of oxidative stress, inflammation, immune activation ensues, which favors carcinogenesis and its progression [63]. For that reason, majority of cancers, especially solid tumours [64], myeloproliferative disorders [65], begin in a setting of oxidative stress and inflammation $[30,66]$.

Further, oxidative stress-induced inflammation and immune activation are thought to cause metabolic dysregulation, persistent activation of anti-apoptotic gene Bcl-2 due to increased ROS as well as the reducing tumour microenvironment [44]. This leads to persistent activation of oncogenes Ras and Myc, tumour suppressor genes p53, RB, BRAC 1 and 2. Likewise, it leads to NF-kB signaling by ROS, increased oxidative stress-induced inflammation and immune activation. This induces metabolic reprogramming and dysregulation causing cells to loss their growth suppression mechanism $[67,68,69,70,71,72]$. ROS also activates pro-inflammatory cytokines, growth factors and Matrix metalloproteinases (MMPs), inducing fibrosis. Notably, activated macrophages, neutrophils express enzymes such as myeloperoxidase (MPO) and NADPH oxidase, which generates ROS [56].

Critical to our understanding of environmental, lifestyle-induced cancers, is acknowledging, appreciating the potential role of pro-inflammatory milieu, pro-carcinogenic properties of carcinogens and their impact in carcinogenesis $[21,23,73]$. It is known that chemical carcinogens contribute to tumourigenesis by modifying the 
tumour microenvironment [47]. As a result, specific changes in the tumour microenvironment can be used as early biomarkers of chemical carcinogens exposure [23].

Significantly, cancer is characterized by seven characteristics or hallmarks;

1. Sustained proliferative signaling,

2. Evading growth suppression,

3. Activating invasion and metastasis,

4. Enabling replicative immortality,

5. Induction of angiogenesis,

6. Resisting cell death

7. Immune evasion and tolerance [30, 59].

By understanding how ROS, oxidative stress-induced inflammation and immune activation interacts with the seven cancer hallmarks, how to target them, identify early biomarkers of carcinogenesis, we will be able to, predict, anticipate and accelerate development of therapeutic targets and preventive strategies. This will have a direct impact on cancer management, help in attainment of United Nations SDG number 3 on ensuring health for all and promotion of wellbeing for all at all ages.

\subsection{Objectives}

To investigate the hypothesis that oxidative stress, inflammation and immune activation is strongly associated with induction of the seven cancer hallmarks, the author reviewed existing literature to identify critical knowledge, research gaps, to stimulate further research interest in the field, accelerate discovery of therapeutic targets and preventive strategies.

The objectives of this systematic review were to:

1. Review the existing literature on oxidative stress, inflammation, immune activation, cancer hallmarks to identify knowledge and research gaps to stimulate further research interest in the field.

2. Exploit the identified knowledge, research gaps to provide new thinking, mechanistic insights, generate new hypothesis, for further research in carcinogenesis, induction of the seven cancer hall marks, to accelerate discovery of new therapeutic targets and preventive strategies.

The author was trying to answer the following review questions whose answers hold the key to understanding carcinogenesis, cancer maintenance, and progression:
1. Whether oxidative stress-induced inflammation, immune activation is involved in induction of the seven cancer hallmarks and thus carcinogenesis?

2. If oxidative stress-induced inflammation, immune activation is involved, how; cause or consequence? And to what extent?

3. Whether targeting oxidative stress-induced inflammation and immune activation signaling can be exploited as therapeutic targets or preventive strategies?

4. Whether selected markers of oxidative stress, inflammation and immune activation can be used as early biomarkers of carcinogenesis?

\section{Materials and Methods}

This was a descriptive review of the existing literature on oxidative stress, inflammation, immune activation and the seven cancer hallmarks. This study was conducted at Kirinyaga University from $10^{\text {th }}$ February 2016 to $25^{\text {th }}$ April, 2017. The inclusion criteria included; only full access articles in English, publications on oxidative stress, inflammation, immune activation, cancer hallmarks. Exclusion criteria included all publication not in English language, not directly associated with the search words; cancer, oxidative stress, inflammation, immune activation, cancer hallmarks, carcinogenesis, involving all the other diseases apart from cancer. The keywords were developed by breaking down the review questions. From the objectives and review questions, a search strategy using different combinations of the key search words was used. The following databases PubMed, Google scholar and ScienceDirect were searched and downloaded at Kirinyaga University using the previously identified keywords. A total of 129 relevant articles out of 8,680,095 hits from the three databases were selected after pearling, critical appraisal to assess the quality, what was analyzed, results obtained, relevance of the downloaded articles to the current study. The articles were reviewed for knowledge, research gaps in cancer, oxidative stress, inflammation, immune activation, therapeutic targets the seven cancer hallmarks. The knowledge, research gaps in the current literature were identified, highlighted, compared and documented. The results of the relevant articles were compared, appraised, analyzed, summarized, presented in the form of summary tables as shown in Tables 1 and 2.

Table 1. Existing studies related to cancer, oxidative stress, inflammation and immune activation

\begin{tabular}{|c|c|c|c|c|}
\hline Databases/Cancer & $\begin{array}{c}\text { Oxidative } \\
\text { stress }\end{array}$ & Inflammation & $\begin{array}{c}\text { Immune } \\
\text { activation }\end{array}$ & $\begin{array}{c}\text { Oxidative stress, inflammation and immune activation as } \\
\text { therapeutic targets }\end{array}$ \\
\hline PubMed & $* 224$ & 12,925 & $* 4424$ & $* 5$ \\
\hline Google Scholar & $2,080,000$ & $3,140,000$ & $2,910,000$ & 139,100 \\
\hline ScienceDirect & 85,169 & 181,344 & 126,315 & $* 589$ \\
\hline Total & $\mathbf{2 , 1 6 5 , 3 9 3}$ & $\mathbf{3 , 3 3 4 , 2 6 9}$ & $\mathbf{3 , 0 4 0 , 7 3 9}$ & $\mathbf{1 3 9 , 6 9 4}$ \\
\hline
\end{tabular}


Table 2. Existing studies related to cancer and the seven cancer hallmarks

\begin{tabular}{|c|c|c|c|c|c|c|c|}
\hline $\begin{array}{c}\text { Databases/Oxidative } \\
\text { stress, Inflammation \& } \\
\text { Immune activation }\end{array}$ & $\begin{array}{c}\text { Proliferative } \\
\text { signaling }\end{array}$ & $\begin{array}{c}\text { Evading } \\
\text { growth } \\
\text { suppression }\end{array}$ & $\begin{array}{c}\text { Resisting } \\
\text { apoptosis }\end{array}$ & $\begin{array}{c}\text { Replicative } \\
\text { immortality }\end{array}$ & $\begin{array}{c}\text { Sustained } \\
\text { angiogenesis }\end{array}$ & $\begin{array}{c}\text { Tissue } \\
\text { invasion \& } \\
\text { Metastasis }\end{array}$ & $\begin{array}{c}\text { Immune } \\
\text { evasion \& } \\
\text { Tolerance }\end{array}$ \\
\hline PubMed & $* 1$ & $* 0$ & $* 0$ & $* 0$ & $* 15$ & $* 1$ & $* 0$ \\
\hline Google Scholar & 24,600 & 19,300 & 135,000 & $* 4,490$ & 32,900 & 31,800 & $* 12,000$ \\
\hline ScienceDirect & $* 105$ & $* 663$ & $* 1,212$ & $* 105$ & $* 6,396$ & $* 2,203$ & $* 403$ \\
\hline Total & $\mathbf{2 4 , 7 0 6}$ & $* \mathbf{1 9 , 9 6 3}$ & $\mathbf{1 3 6 , 2 1 2}$ & $* \mathbf{4 , 5 9 5}$ & $\mathbf{3 9 , 3 1 1}$ & $\mathbf{3 4 , 0 0 4}$ & $* \mathbf{1 2 , 4 0 3}$ \\
\hline
\end{tabular}

\section{Results}

Table 1 summarizes existing studies on cancer, oxidative stress, inflammation, immune activation. The areas of cancer, oxidative stress, inflammation, immune activation are well studied. However, few studies exist on oxidative stress, inflammation, immune activation as therapeutic targets. Future research should focus on targeting them as therapeutic targets. $*=$ indicates not widely published area. Only 5 Downloads were gotten on oxidative stress, inflammation and immune activation as cancer therapeutic targets in PubMed

Table 2 summarizes existing studies on oxidative stress, inflammation, immune activation and the seven cancer hallmarks. Generally, the area of oxidative stress, inflammation, immune activation and cancer hallmarks is not widely studied, indicating the potential for future research this field holds $*=$ Indicates not well published area. The area of cancer hallmarks is not exhaustively studied. No single study on cancer, evading growth suppression, resisting apoptosis, replicative immortality immune evasion and tolerance was found in the PubMed database, indicating a field where more research should focus on.

\section{Discussion}

Based on the objectives and review questions of the descriptive survey, different combinations of the search words, a total of $8,680,095$ hits for studies relating to cancer, oxidative stress, inflammation, immune activations independently and as therapeutic targets were gotten. Likewise, 271,194 hits relating to cancer and the seven cancer hallmarks were obtained. The downloaded hits were appraised critically, assessed for relevance to the survey, quality, methods, results, after which 129 articles were selected. The articles were reviewed to identify knowledge, research gaps, results compared and presented in form of summary tables as shown in Table 1 and 2 .

According to the results (Table 1), the field of cancer, oxidative stress, inflammation, immune activation is well researched with a lot of published literature on the same. However, as therapeutic targets, these areas have not been adequately investigated. Out of the total 8,680,095 hits, only 139,694 hits related to oxidative stress, inflammation and immune activation as therapeutic targets as shown in Table 1. Notably, cancer hallmarks have not been exhaustively studied, specifically, proliferative signaling, tissues invasion, metastasis as shown in Table 2. Of concern, there were only $4,595,12,403,19,963$ hits related to oxidative stress, inflammation, immune activation and replicative immortality, immune evasion, evading of growth suppression respectively out of the total 271,194 hits as indicated in Table 2. Consequently, the role of oxidative stress, inflammation, immune activation in induction of the cancer hallmarks is not clearly defined. This makes these areas potential research themes worth consideration in prospective studies. Hence, future studies should focus on the induction of the cancer hallmarks with special emphasis on the role (whether causal or consequence) of ROS, oxidative stress, inflammation, immune activation and how to target them as therapeutic interventions.

That ROS are constitutively generated during cellular metabolism in mitochondrial and in response to a variety of environmental stimuli including ultraviolet (UV), ionizing radiation, (IV) chemicals, hyperoxia etc. is clear. In addition, the importance of chronic oxidative stress, inflammation, immune activation in carcinogenesis is well documented. However, their role in inducing the seven cancer hallmarks is not clearly delineated. Therefore, specific studies focusing primarily on the causal association, if at all, between oxidative stress, inflammation, immune activation and the seven cancer hallmarks are required.

\subsection{Identified Knowledge and Research Gaps; Limitation of the Current Cancer Therapies}

Recovery from cancer depends on the type, the stage when diagnosed, the trait or cells/tissue affected level, nature of the treatment, emotional and physical health of the patient $[14,15]$. However, the current cancer therapies are limited in their action and not $100 \%$ effective, thus do not achieve a complete remission. As a consequence, majority of cancers have a five-year survival rate. In addition, they target single signaling pathways, indiscriminately kill tumour cells and in the process normal cells, hence the serious adverse effects associated with chemotherapy. The overlap and multifunction of signaling pathways, makes targeting them a challenging task $[73,74,75]$. Likewise, some of the interventions target downstream events, which occur much later such as DNA damage repair, eliminating tumour cells or excising mutations. Furthermore, majority of the interventions overlook early events of carcinogenesis. Of major concern, most of the cancers are diagnosed at 
advanced stages, offering little chance of survival as mentioned earlier $[9,10]$.

In view of this and considering the multi-etiological nature of cancer, future therapies call for a safe, effective and multi-target approach.

\subsection{The Seven Cancer Hallmarks and How to Target Them}

According to the results, though oxidative stress-induced inflammation and immune activation are thought to interact with all the seven cancer hallmarks, exact mechanisms through which this leads to initiation, maintenance, cancer progression as well as induction of the seven cancer hallmarks is not clear. In this review, the author proposes how ROS, oxidative stress, inflammation, immune activation may interact to induce the seven cancer hallmarks and how to target them in future research.

\section{Sustained Proliferative Signaling, Evading Growth Suppression, Resisting Cell Death}

The heightened, persistent ROS in the mitochondria due to increased cellular energy demands, the ensuing oxidative stress-induced inflammation and immune activation are thought to cause a sustained, excessive NF-kB and Ras signaling. Subsequently, this leads to mitochondria DNA (mtDNA) damage [76] that increases the cellular energy demands to compensate for the mitochondrial damage, meet increased energy demands to clear the ROS and oxidative stress $[76,77,78]$. Similarly, metabolic reprogramming ensues, which is characterized by increased glycolysis (glycolytic flux) [34]. This, in concurrence with the ROS activates proto-oncogenes (Ras, c-Myc) [68, 70, 79, 80] tumour suppressor genes (p53, RB, BRCA 1 \& 2) [67] causes "driver mutations" [81], telomere shortening (increased telomerase activity), decreased cellular senescence, [82]. Significantly, increased growth factors signaling resulting from increased ROS, persistent NF-kB activation, is known to antagonize apoptotic signaling, probably as a cellular adaptation mechanism $[79,81,82,83$, 84]. Accordingly, this leads to; sustained proliferative signaling $[85,86]$, evading of growth suppression, acquisition of replicative immortality and resistance to cell death as a preservation mechanism [81] or ROS insult and activation of anti-apoptotic gene $\mathrm{Bcl}-2$ [86,87]. The increased competition for nutrients by the rapidly dividing tumorous cells is thought to cause the clonal selection characteristic of many cancers probably as an adaptation to the competition [44].

Amelioration of the effects of ROS, oxidative stress-induce inflammation and immune activation by modulation of excessive oxidative phosphorylation, thus decreasing ROS generation by use of immune modulators such as selected antioxidants, inhibitors of oxidative phosphorylation inhibitors (e.g. Metformin), Nonsteroidal anti-inflammatory drugs (NSAIDs) as a combination, might modulate the excessive NF-kB signaling, prevent mitochondria, mtDNA damage, eliminate the need for increased cellular energy demands, restore normal cellular metabolism, preventing induction of replicative immortality, resistance to apoptosis, stop induction of the 'driver mutations' in proto-oncogenes and tumour suppressor genes. Furthermore, modulation of ROS, oxidative stress-induced inflammation, immune activation will prevent induction of immune suppressive T-regs and preserve the integrity of cytotoxic T-cells (CD8T-cells), which will identify, eliminate tumour cells restoring a normal immune surveillance function.

\section{Activating Tumour Invasion and Metastasis}

The increased and persistent ROS, oxidative stress, competition for oxygen and nutrients by the rapidly dividing tumour cells leads to hypoxia and clonal selection, which is a common characteristic of cancers [73, 88]. Likewise, it leads to increased secretion of pro-inflammatory cytokines by stromal cells in the tumour microenvironment. This leads to induction of Matrix metalloproteinases (MMPs) [89], which degrades the extracellular matrix (ECM), gap junctions and contact inhibition [90, 91, 92]. This in addition to competition for $\mathrm{O}_{2}$ and nutrients activate tumour invasion and metastasis. Furthermore, the reducing environment of the tumour microenvironment due to increased ROS, oxidative stress also contributes events leading to tumour evasion and metastasis. Activation of the innate immunity (neutrophils and macrophages) by adaptive immunity produces an enhanced respiratory burst leading increased production of pro-inflammatory cytokines and ROS leading to a vicious cycle of oxidative stress, inflammation, immune activation and increased production of MMPs [93]. Consequently, modulating ROS, oxidative stress-induced inflammation and immune activation will reverse the hypoxia, production of MMPs and pro-inflammatory cytokines especially in the tumour microenvironment; modify the reducing environment and the adverse effects.

\section{Enabling Replicative Immortality}

The increased ROS and oxidative stress signaling of NF-kB, Ras, p53, anti-apoptotic Bcl-2, metabolic reprogramming and dysregulation, telomere shortening and decreased cellular senescence $[82,94]$ are thought to cause resistance to normal apoptosis or enabling replicative immortality probably as an adaptation to the increased and persistent ROS attack $[44,30]$. By modulating the increased $\mathrm{NF}-\mathrm{kB}$, we can regulate Ras and Bcl-2 signaling, increase telomerase activity and promote senescence.

\section{Inducing Angiogenesis}

In order to meet the increased oxygen and nutrients demands, the rapidly dividing cells requires new blood vessels, hence need for angiogenesis $[45,95]$. The increased and persistent ROS, oxidative stress, metabolic 
reprogramming, low oxygen from oxidative stress (hypoxia), increased cellular energy demands by the rapidly dividing cells, ensuing competition for the inadequate oxygen, nutrients leads to production of hypoxia inducible factor I (HIF-I), tissue factor (TF) and VEGF [85,96-98] by endothelia cells, which initiates angiogenesis. The competition leads also to clonal selection, which characterizes cancers [81]. Therefore, modulating ROS and oxidative stress signaling might reduce hypoxia, cellular energy demands, competition for oxygen $\left(\mathrm{O}_{2}\right)$, nutrients, induction of HIF-I, TF, VEGF, thus angiogenesis and clonal selection.

\section{Immune Evasion and Tolerance}

The increased and persistent ROS, oxidative stress-induced inflammation and immune activation are implicated in the sustained NF-kB [99], Ras signaling [69], down regulation of MHC I in CD8 T-cells [100], thus cannot recognize and eliminate tumour cells. Furthermore, increased oxidative stress is thought to induce the immunosuppressive T-regs [101]. T-regs suppresses other immune cells via production of IL-10. Therefore, modulating ROS, oxidative stress, inflammation, immune activation will reduce the excessive NF-kB signaling, reverse the adverse effects of the signaling, preserve integrity of CD8 T-cell enabling them to recognize and eliminate tumour cells.

In addition, it will prevent induction of T-regs, preserving the function of other immune cells and the immune system. Furthermore, it will reverse hypoxia, production of MMPs, pro-inflammatory cytokines especially in the tumour microenvironment; modify the reducing environment and the adverse effects. Specifically, modulating the increased $\mathrm{NF}-\mathrm{kB}$, signaling will regulate Ras, Bcl-2 signaling, decrease the telomerase activity and increase cellular senescence. In addition, the modulation will reduce hypoxia, decrease cellular energy demands, thus decrease competition for oxygen, nutrients, induction of HIF-I, TF, VEGF and clonal selection. Lastly, the modulation will preserve the integrity of CD8 T-cell enabling them to recognize tumour cells, eliminating them, preventing induction of T-regs, preserving the function of other immune cells and the overall immune system surveillance.

The author believes and is supported by various previous studies $[20,44,21,30,31,32]$ that oxidative stress, inflammation, immune activation conspires to produce carcinogenic synergies, that are regrettably disregarded by the existing risk assessment methods. Accordingly, exposure to immune disruptors, the common chemical, environmental carcinogens discussed previously in the introduction, cancer-inducing infectious biological agents (bacteria, viruses, aflatoxin), chronic inflammatory conditions (HIV/AIDS, diabetes mellitus), induces a certain degree of oxidative stress, DNA damage, initial focal proliferative lesions, which leads to inflammation, immune activation, recruitment of inflammatory cells, with generation of more ROS, pro-inflammatory cytokines, immune activation, leading to a vicious cycle of oxidative stress and inflammation [57,102]. The oxidative stress-induced inflammatory milieu leads to genomic instability, DNA damage, increased generation of pro-inflammatory cytokines, more oxidative stress, inflammation, immune activation, increased cellular energy demands, leading to metabolic reprogramming, hypoxia, competition for oxygen, nutrients, induction of "driver mutations" in proto-oncogenes and tumour suppressor genes [81]. All these events conspire to ultimately induce the characteristic seven cancer hallmarks. Other consequences include; damage of CD8 T- lymphocytes by downregulation of MHC I, hence cannot recognize, destroy tumour cells and induction of the immunosuppressive T-regs (immune evasion \& tolerance). Likewise, amelioration of the oxidative stress, inflammation and immune activation is the first step in preventing and stopping carcinogenesis.

Previous strategies to target the cancer hallmarks such as moderation of immune action, metabolic reprogramming have been tried and reported [103,104]. Of special importance, Metformin, an anti-diabetic, has been shown to inhibit mitochondrial respiration, mitochondrial respiratory chain (complex), neoplastic angiogenesis, mammalian target of rapamycin (mTOR) complex 1 signaling, modulate cancer inflammatory microenvironment, promote both caspase-dependent and independent apoptosis, suppress glucose production influx of glucagon from glycolysis and impairment of oxidative phosphorylation thus preventing production of ROS [105].

Notably, regular use of small doses of aspirin and other NSAIDS has been reported to reduce the risk of getting cancers $[106,107]$. Evidence that immune moderation is effective in cancer therapy is provided by the effective use of alpha Interferons (IFNs) as the first line of treatment in management of Chronic Myeloid Leukemia (CML), which replaces the use of cytotoxic drugs (e.g. Hydroxyurea and Busulfan) in patients not eligible for bone marrow transplantation (i.e. those over 50 years) [108].

The future challenge is designing a combined formulation targeting oxidative stress, inflammation, immune activation signaling and an effective delivery system of the same. However, more studies on the role of oxidative stress, inflammation, immune activation in carcinogenesis, cancer maintenance, its progression, hallmarks are required to strengthen the hypothesis. In addition, preventive measures such as active lifestyle, weight loss, stress reduction, diets rich in anti-inflammatories, anti-bacterial, modulators of oxidative stress and immune activation, discouraging plastic packaging of foods, drinks, increased intake of fruits, vegetables, reduction of alcohol consumption, tobacco smoking, chewing, frequent screening for cancers, early diagnosis, treatment of cancer and chronic inflammatory conditions are proposed. 


\subsection{Potential Oxidative Stress, Inflammation and Immune Activation Early Biomarkers of Cancer}

Most cancers exhibit long latency period, hence early biomarkers to detect them during this period as well as understanding the primary changes within the tumour microenvironment will be essential in their timely diagnosis, treatment and management [20]. Significantly, the causal association of genomic instability, inflammation in cancer, metabolic reprogramming, immune evasion, tolerance as enablers, makes them ideal early biomarkers [61]. Early potential biomarkers of cancer can either be direct or indirect. The direct early biomarkers include; upregulation or overexpression of genes regulating metabolism, NF-kB signaling, anti-apoptotic genes (Bcl-2, Bcl-X, Bcl-W, MCL-1, BCL-B), down regulation of pro-apoptotic genes (Bax, Bak, Bik, Bad, Bim, HRK) [109, 110]. Other direct biomarkers include; upregulation or altered gene expression of oncogenes (Ras, c-Myc), tumour suppressor genes (p53, $\mathrm{RB}$ ), hypoxia-associated transcription, growth factors (HIF-1, TF, VEGF) [111], MHC molecules, downregulation of adhesive molecules, growth factors and their surface receptors) in histologically normal tissues.

Consequently, potential early markers of dysregulated metabolism includes; increased mtDNA, increased lactate dehydrogenase (LDH), which catalysis the reversible $\mathrm{NAD}^{+} / \mathrm{NADH}$-dependent conversion of pyruvate, lactate and an important source for $\mathrm{NAD}^{+}$required for glycolytic flux [112,113]. Similarly, increased or mutations in isocitrate dehydrogenase (IDH) from cancer-associated mutations, which promote on cometabolites formation [114, 115], increased fumarate hydratase [114] phosphofructokinase (PFK), a major irreversible rate-controlling step of glycolysis are other possible biomarkers [115, 116,117,118]. Early biomarkers of dysregulated metabolism include; increased AcetylCoA, lipogenesis for supporting membrane biogenesis, which is characteristic of many aggressive cancers $[119,120]$. In addition, DNA damage products such as 8-oxo-2'deoxyguanosine have been used previously as early biomarkers of carcinogenesis [121]. Likewise, over expression of VEGF [95], and MMPs can be considered as potential early biomarker of carcinogenesis [122].

Indirect early biomarkers include; increased chronic inflammation (Fibrinogen, D-Dimers), immune activation markers (CD25, CD69, CD38), increased fatty acid metabolism (lipogenesis), decreased antioxidant defenses (Nuclear factor erythroid 2 related factor 2 (Nrf2), reduced glutathione (GSH), glutathione peroxidase (GPx), Catalase, superoxide dismutase (SOD)), inhibition of caspase (caspase 3) activation, down-regulation of $\mathrm{MHC} \mathrm{I}$ in $\mathrm{CD} 8$ T-lymphocytes and increased T-regs among other $[123,124]$. More importantly, specific alterations in the tumour microenvironment, bone marrow such as increased oxidative metabolism (enhanced respiratory burst and pentose phosphate pathway [PPP] activation), TP53-induced glycolysis, apoptotic regulator (TIGAR), chronic inflammation, immune activation markers, activated stroma and other cells, overexpression of growth factors (IL-I, IL-2, SCF, IL-3, IL-5, IL-6, VGEF, CSF-GM, CSF-G, hepatocyte growth factor [produced by tumour-associated fibroblasts] [125] and CSF-M), adhesive molecules, cell surface receptors, increased hypoxia, hypoxia inducible factor-I (HIF-I) genes, VEGF can be used to predict or for early diagnoses of cancers $[126,127]$. Other indirect potential early biomarkers include; increased MMPs, transcription factors regulating conversion of epithelial to mesenchymal Transition (EMT) such as snail, slug, and twist [128,129], genes are important early biomarkers of cancer.

Over the last few years, there has been an explosion of cancer information, which has led to better understanding of cancer aetiology, risk factors, chemical, environmental carcinogens, role of diet, lifestyle, obesity, stressful lifestyle, chronic inflammatory conditions such as HIV/AIDS in carcinogenesis. Recently, recognition of the important role oxidative stress, inflammation and immune activation plays in carcinogenesis, cancer maintenance, progression presents promising future novel therapeutic targets and preventive strategies.

\section{Conclusions}

Oxidative stress, inflammation, immune activation are important participants in carcinogenesis, hence targeting their signaling, activation will be therapeutic and preventive. The multifactorial etiology of cancer necessitates a combined multifactorial approach targeting all the seven cancer hallmarks. This approach represents an important strategy for cancer prevention and treatment. For that reason, targeting oxidative stress-induced inflammation and immune activation presents a radical cancer therapeutic and preventive approach. In addition, modulation of ROS, oxidative stress-induced inflammation and immune activation will prevent induction of immune suppressive T-regs and preserve the integrity of cytotoxic T-cells (CD8), which will eliminate cancerous cells.

\section{REFERENCES}

[1] WHO. 2017 Cancer, Fact sheets. http://www.who.int/mediacentre/factsheets/fs297/en/. Downloaded on $20^{\text {th }}$ October, 2016.

[2] P., Malloy, J., Boit, J. M., Allison Tarus, B., Ferrell \& Z. Ali. Providing palliative care to patients with cancer: Addressing the needs in Kenya. Asia-Pacific Journal of Oncology Nursing, 4(1), 45, 2017.

[3] Kenya Network of Cancer organizations (KNCO). 2016. https://kenyacancernetwork.wordpress.com/kenya-cancer-fact $\mathrm{s} /$. Downloaded on $20^{\text {th }}$ October, 2016.

[4] SF. Cancer Burden in Kenya. 2010. http://www-pub.iaea.org/ 
$\mathrm{mtcd} /$ meetings/PDFplus/2010/cn189/cn189Presentations/SF2 010_Kenya.pdf. Downloaded on $20^{\text {th }}$ November, 2016.

[5] American Cancer Society (ACS). Cancers that develop in Children. 2016.

https://www.cancer.org/cancer/cancer-in-children/types-of-ch ildhood-cancers.html Downloaded on 10th January, 2017.

[6] CDC. Human Papillomavirus Vaccination: Recommendations of the Advisory Committee on Immunization Practices (ACIP). 2014

https://www.cdc.gov/mmwr/preview/mmwrhtml/rr6305a1.ht $\mathrm{ml}$. Downloaded on $10^{\text {th }}$ January, 2017.

[7] J. R. Sandfort \& A. Pleasant. Knowledge, attitudes, and informational behaviors of college students in regard to the human papillomavirus. Journal of American College Health, 58(2), 141-149. 2009.

[8] A. L. Friedman \& H. Shepeard. Exploring the knowledge, attitudes, beliefs, and communication preferences of the general public regarding HPV findings from CDC focus group research and implications for practice. Health Education \& Behavior, 34(3), 471-485. 2007.

[9] J. F. Onyango \& I. M. Macharia. Delays in diagnosis, referral and management of head and neck cancer presenting at Kenyatta National Hospital, Nairobi. East African medical journal, 83(4), 85-91. 2006.

[10] Kenya Hospices and Palliative Care Association (KEHPCA). Annual Report. 2014. Available from: http://www.kehpca.org/wp-content/uploads/Annual-Report-2 014_draft.pdf. Downloaded on 5th October, 2016.

[11] AACR. 2017. What is Cancer? https://www.aacrfoundation.org/Pages/what-is-cancer.aspx. Downloaded on $10^{\text {th }}$ October, 2016.

[12] S. B. Edge \& C. C. Compton. The American Joint Committee on Cancer: the 7th edition of the AJCC cancer staging manual and the future of TNM. Annals of surgical oncology, 17(6), 1471-1474. 2010.

[13] John Hopkins Medicine. Types of Cancer. 2017. http://www. hopkinsmedicine.org/kimmel_cancer_center/types_cancer/m ultiple_myeloma.html. Downloaded on $10^{\text {th }}$ October, 2016.

[14] L. Witek-Janusek, K. Albuquerque, K. R. C. ChroniakChroniak, R., Durazo-Arvizu \& H. L. Mathews. Effect of mindfulness based stress reduction on immune function, quality of life and coping in women newly diagnosed with early stage breast cancer. Brain, behavior, and immunity, 22(6), 969-981. 2008.

[15] S. M. Levy \& B. D. Wise. Psychosocial risk factors, natural immunity, and cancer progression: Implications for intervention. Current Psychology, 6(3), 229-243. 1987.

[16] R. Doll et al. The causes of cancer: quantitative estimates of avoidable risks of cancer in the United States today. J. Natl. Cancer Inst., 66, 1191-1308. 1981

[17] G.A. Colditz et al. Preventability of cancer: the relative contributions of biologic and social and physical environmental determinants of cancer mortality. Annu. Rev. Public Health, 33, 137-156. 2012

[18] J. Malhotra. Molecular and genetic epidemiology of cancer in low- and medium-income countries. Ann. Glob. Health, 80, 418-425. 2014
[19] C. McGuinn, M. B. Geyer, Z. Jin, J. H. Garvin P. Satwani M. B. Bradley \& C. van de Ven, Pilot trial of risk - adapted cyclophosphamide intensity based conditioning and HLA matched sibling and unrelated cord blood stem cell transplantation in newly diagnosed pediatric and adolescent recipients with acquired severe aplastic anemia. Pediatric blood \& cancer, 61(7), 1289-1294. 2014.

[20] W.H. Goodson. Assessing the carcinogenic potential of low-dose exposures to chemical mixtures in the environment: the challenge ahead, Carcinogenesis, 36, S254-S296. 2015.

[21] P.A. Thompson. Environmental immune disruptors, inflammation and cancer risk, Carcinogenesis, 36, S232-253.2015.

[22] D.M. Parkin et al. The fraction of cancer attributable to lifestyle and environmental factors in the UK in 2010. Br. J. Cancer, 105(Suppl. 2), S77-S81. 2011

[23] S.C. Casey et al. The effects of environmental chemicals on tumour microenvironment. Carcinogenesis, 36, S160-S183. 2015.

[24] L.E. Thueson, et al. In vitro exposure to the herbicide atrazine inhibits $\mathrm{T}$ cell activation, proliferation, and cytokine production and significantly increases the frequency of Foxp3+ regulatory T cells. Toxicol. Sci., 143, 418-429. 2015.

[25] K.B. Narayanan et al. Disruptive environmental chemicals and cellular mechanisms that confer resistance to cell death, Carcinogenesis, 36, S89 -S110, 2015.

[26] S.A.S. Langie et al. Causes of genome instability: the effect of low dose chemical exposures in modern society, Carcinogenesis, 36, S61-S88, 2015.

[27] C.Y. Hsiang et al. Acetaldehyde induces matrix metalloproteinase- 9 gene expression via nuclear factor-kappaB and activator protein 1 signaling pathways in human hepatocellular carcinoma cells: association with the invasive potential. Toxicol. Lett., 171, 78-86. 2007

[28] S.Z. Ding et al. Epithelial-mesenchymal transition during oncogenic transformation induced by hexavalent chromium involves reactive oxygen species-dependent mechanism in lung epithelial cells. Toxicol. Appl. Pharmacol., 269, 61-71. 2013

[29] X.Wang et al. Arsenic and chromium in drinking water promote tumorigenesis in a mouse colitis-associated colorectal cancer model and the potential mechanism is ROS-mediated Wnt/beta-catenin signaling pathway. Toxicol. Appl. Pharmacol., 262, 11-21. 2012

[30] F. Colotta et al. Cancer-related inflammation, the seventh hallmark of cancer: link to genetic instability. Carcinogenesis, 30, 1073-1081. 2009.

[31] V. Vallyathan, X. Shi \& V. Castranova. Reactive oxygen species: their relation to pneumoconiosis and carcinogenesis. Environmental health perspectives, 106(Suppl. 5), 1151. 1998.

[32] N. Azad, Y. Rojanasakul, \& V. Vallyathan. Inflammation and lung cancer: roles of reactive oxygen/nitrogen species. Journal of Toxicology and Environmental Health, Part B, 11(1), 1-15. 2008.

[33] R. Truhaut. Recent progress in the evaluation of the dangers of chemical carcinogens. Journal de pharmacie de Belgique, 
45(2), 131-140. 1989.

[34] T. Fiaschi et al. Oxidative stress, tumour microenvironment and metabolic reprogramming, a diabolical liason. Int. J. Cell Biol., 2012, 762-825. 2012.

[35] P.C. Hanawalt, Subpathways of nucleotide excision repair and their regulation. Oncogene, 21, 8949-8956. 2002.

[36] S. Shishodia, et al. Curcumin (diferuloylmethane) downregulates cigarette smoke-induced NF-kappaB activation through inhibition of IkappaBalpha kinase in human lung epithelial cells: correlation with suppression of COX-2, MMP-9 and cyclin D1. Carcinogenesis, 24, 1269-1279. 2003.

[37] S.K. Srivastava, et al. Cell cycle arrest, apoptosis induction and inhibition of nuclear factor kappa B activation in anti-proliferative activity of benzyl isothiocyanate against human pancreatic cancer cells. Carcinogenesis, 25, 1701-1709. 2004.

[38] R.J. Gillies, et al. Hypoxia and adaptive landscapes in the evolution of carcinogenesis. Cancer Metastasis Rev., 26, 311-317. 2007.

[39] S. Radice et al. Adaptation to oxidative stress: effects of vinclozolin and iprodione on the HepG2 cell line. Toxicology, 129, 183-191.1998.

[40] AB. Motta. Mechanisms involved in Metformin action in the treatment of Polycystic Ovary Syndrome. Curr Pharm Des. 15(26): 3074-7. 2009.

[41] S.C. Laws et al. Chlorotriazine herbicides and metabolites activate an ACTH-dependent release of corticosterone in male Wistar rats. Toxicol. Sci., 112, 78-87. 2009.

[42] T. Calandra, et al. MIF as a glucocorticoid-induced modulator of cytokine production. Nature, 377, 68-71. 1995

[43] R. Bucala. MIF rediscovered: cytokine, pituitary hormone and glucocorticoid-induced regulator of the immune response. FASEB J., 10, 1607-1613. 1996

[44] R.B. Robey et al. Metabolic reprogramming and dysregulated metabolism: cause, consequence and/or enabler of environment carcinogenesis? Carcinogenesis, 36, S160-S231. 2015.

[45] D. Hanahan et al. The hallmarks of cancer. Cell, 100, 57-70. 2000

[46] F. Balkwill et al. Inflammation and cancer: back to Virchow? Lancet, 357, 539-545. 2001

[47] S. Laconi et al. A growth-constrained environment drives tumor progression invivo. Proc. Natl Acad. Sci. USA, 98, 7806-7811. 2001

[48] J.P. Hindley, et al. Analysis of the T-cell receptor repertoires of tumor-infiltrating conventional and regulatory $\mathrm{T}$ cells reveals no evidence for conversion in carcinogen-induced tumors. Cancer Res., 71, 736-746. 2011

[49] R.L. Ferris, et al. Human leukocyte antigen (HLA) class I defects in head and neck cancer: molecular mechanisms and clinical significance. Immunol. Res., 33, 113-133. 2005

[50] F.Cavallo, et al. 2011: the immune hallmarks of cancer. Cancer Immunol. Immunother., 60, 319-326. 2011
[51] T.L. Whiteside. Immune suppression in cancer: effects on immune cells, mechanisms and future therapeutic intervention. Semin. Cancer Biol., 16, 3-15. 2006

[52] M. Sugaya. Chemokines and cutaneous lymphoma. J. Dermatol. Sci., 59, 81-85. 2010

[53] K.L. Knutson et al. Immunoediting of cancers may lead to epithelial to mesenchymal transition. J. Immunol., 177, 1526-1533. 2006

[54] A.F. Olumi, et al. Carcinoma-associated fibroblasts direct tumor progression of initiated human prostatic epithelium. Cancer Res., 59, 5002-5011. 1999

[55] A.M. Briones, \& R.M. Touyz. Oxidative stress and Hypertension: Current Concepts. $9^{+}$Curr Hypertens Rep, 12: 135-142. 2010.

[56] A.R Weseler, \& A. Bast. Oxidative stress and vascular Function: Implications for Pharmacological Treatments. Curr Hypertens Rep, 12: 154-161. 2010.

[57] M. Khatami. Unresolved inflammation: 'immune tsunami' or erosion of integrity in immune-privileged and immune-responsive tissues and acute and chronic inflammatory diseases or cancer. Expert Opin. Biol. Ther., 11, 1419-1432. 2011

[58] P. L., da Costa P. Sirois I. F. Tannock \& R. Chammas. The role of kinin receptors in cancer and therapeutic opportunities. Cancer letters, 345(1), 27-38. (2014).

[59] L. Danelli, B. Frossi, G. Gri, F. Mion, C. Guarnotta, L. Bongiovanni \& U. Blank, Mast cells boost myeloid-derived suppressor cell activity and contribute to the development of tumor-favoring microenvironment. Cancer immunology research, 3(1), 85-95. 2015.

[60] M.J. Grimm, R.R. Vethanayagam, N.G. Almyroudis, C. G. Dennis, A.N.H. Khan, A. C.D'Auria \& T.M. Hohl Monocyte-and macrophage-targeted NADPH oxidase mediates antifungal host defense and regulation of acute inflammation in mice. The Journal of Immunology, 190(8), 4175-4184. (2013).

[61] D. Hanahah, et al. Hallmarks of cancer: the next generation. Cell, 144, 646-674. 2011.

[62] B. Loaiza-Ruiz, et al. Understanding cancer stem cell: role of NF-KB in the chemotherapy resistance. In Pandalai, S.G. (ed.) In Recent Research Developments in Cancer. Vol. 9. Transworld Research Network, Kerala, India, pp. 25-50. 2011.

[63] A. M. Knaapen, N. Güngör, R. P. Schins, P. J. Borm \& F. J. Van Schooten Neutrophils and respiratory tract DNA damage and mutagenesis: a review. Mutagenesis, 21(4), 225-236. 2006.

[64] L.B. Meira, et al. DNA damage induced by chronic inflammation contributes to colon carcinogenesis in mice. J. Clin. Invest., 118, 2516-2525. 2008.

[65] H.C. Hasselbalch. Chronic inflammation as a promotor of mutagenesis in essential thrombocythemia, polycythemia vera and myelofibrosis. A human inflammation model for cancer development? Leuk. Res., 37, 214-220. 2013.

[66] J.S. Duffield et al. Host responses in tissue repair and fibrosis. Annu. Rev. Pathol., 8, 241-276. 2013. 
[67] M. Hollstein, et al. P53 mutations in human cancers. Science, 253, 49-53. 1991.

[68] J.W. Jang et al. Iso-form-specific Ras activation and oncogene dependence during MYC and Wnt-induced mammary tumourigenesis. Mol. Cell. Biol., 26, 8109-8121. 2006.

[69] D.M. Munox et al. Loss of p53 cooperates with K-ras activation to induce glioma formation in a region-independent manner. Glia, 61, 1862-1872. 2013.

[70] M.A. Pierotti, et al. (2003). Mechanisms of oncogene activation. Kufe DW, Polock RE, Weichselbaum RR and et al. Holland- Frei Cancer Medicine 6th. Hamilton (ON), BC Decker. 2003.

[71] F. Mazzei, et al. Role of MUTYH in human cancer. Mutat. Res., 743-744, 33-43. 2013.

[72] A. Sancar et al. Excision repair in mammalian cells. J. Bio. Chem., 270, 15915-15918. 1995.

[73] L. Li, et al. Non-monotonic dose-response relationship in steroid hormone receptor-mediated gene expression. J. Mol. Endocrinol., 38, 569-585. 2007.

[74] Y. Lazebnik. What are the hallmarks of cancer? Nat. Rev. Cancer, 10, 232-233. 2010.

[75] S.L. Floor. et al. Hallmarks of cancer: of all cancer cells, all the time? Trends Mol. Med., 18, 509-515. 2012.

[76] J. Kravchenko et al. Chemical compounds from anthropogenic environment and immune evasion mechanisms: potential interactions, Carcinogenesis, 36, S111-S127.2015.

[77] S.M. Hou, et al. The XPD variant alleles are associated with increased aromatic DNA adduct level and lung cancer risk. Carcinogenesis, 23, 599-603. 2002.

[78] R.J. DeBerardinis, et al. The biology of cancer: metabolic reprogramming fuels cell growth and proliferation. Cell Metab., 7, 11-20. 2008.

[79] R.B. Robey et al. Mitochondrial hexokinases, novel mediators of the antiapoptotic effects of growth factors and Akt. Oncogene, 25, 4683-4696. 2006.

[80] J.J. Oudejans et al. Differential activation of ras genes by point mutation in human colon cancer with metastases to either lung or liver. Int. J. Cancer, 49, 875-879. 1991.

[81] A. Carnero. et al. Disruptive chemicals, senescence and immortality, Carcinogenesis, 36, S19 - S37. 2015.

[82] J.W. Shay et al. Hallmarks of senescence in carcinogenesis and cancer therapy. Oncogene, 23, 2919-2933. 2004.

[83] R.B. Robey et al. Mitochondrial hexokinases: guardians of the mitochondria. Cell Cycle, 4, 654-658. 2005.

[84] J.L. Andersen et al. The tangled circuitry of metabolism and apoptosis. Mol. Cell, 49, 399-410. 2013.

[85] S.J. Ralph et al. The causes of cancer revisited: "mitochondrial malignancy" and ROS-induced oncogenic transformation - why mitochondria are targets for cancer therapy. Mol. Aspects Med., 31, 145-170. 2010.

[86] Taguchi, K., Motohashi, H., \& Yamamoto, M. (2011). Molecular mechanisms of the Keap1-Nrf2 pathway in stress response and cancer evolution. Genes to Cells, 16(2), 123-140.

[87] L. Ouyang et al. Programmed cell death pathways in cancer: a review of apoptosis, autophagy and programmed necrosis. Cell Prolif., 45, 487-498. 2012.

[88] K., Degenhardt, R. Mathew, B. Beaudoin, K. Bray, D., Anderson, G. Chen \& D. A. Nelson. Autophagy promotes tumor cell survival and restricts necrosis, inflammation, and tumorigenesis. Cancer cell, 10(1), 51-64. 2006.

[89] H. Birkedal-Hansen et al. Matrix metalloproteinases: a review. Crit. Rev. Oral Biol. Med., 4, 197-250. 1993.

[90] L. Cronier et al. Gap junctions and cancer: new functions for an old story. Antioxid. Redox Signal., 11, 323-338. 2009.

[91] C.C. Naus et al. Implications and challenges of connexin connections to cancer. Nat. Rev. Cancer, 10, 435-441. 2010.

[92] L.A. Shuman Moss et al. Matrix metalloproteinases: changing roles in tumor progression and metastasis. Am. J. Pathol., 181, 1895-1899. 2012

[93] D. Radisky et al. Raclb and reactive oxygen species mediate MMP-3-induced EMT and genomic instability. Nature, 436, 123-127. 2005.

[94] J. Campisi. Cellular senescence: putting the paradoxes in perspective. Curr. Opin. Genet. Dev., 21, 107-112. .2011.

[95] P. Carmeliet et al. Molecular mechanisms and clinical applications of angiogenesis. Nature, 473, 298-307. 2011.

[96] M. Ushio-Fukai et al. Reactive oxygen species and angiogenesis: NADPH oxidase as target for cancer therapy. Cancer Lett., 266, 37-52. 2008.

[97] M. Freitas et al. Nickel induces apoptosis in human neutrophils. Biometals, 26, 13-21. 2013.

[98] J. Li, et al. Nickel compounds act through phosphatidylinositol- 3-kinase/Akt-dependent, p70 (S6k)-independent pathway to induce hypoxia inducible factor transactivation and Cap43 expression in mouse epidermal Cl41 cells. Cancer Res., 64, 94-101. 2004.

[99] S.P. Gabbita et al. Redox regulatory mechanisms of cellular signal transduction. Arch. Biochem. Biophys., 376, 1-13. 2000 .

[100] N.A. Karrow et al. Oral exposure to atrazine modulates cell-mediated immune function and decreases host resistance to the $\mathrm{B} 16 \mathrm{~F} 10$ tumor model in female $\mathrm{B} 6 \mathrm{C} 3 \mathrm{~F} 1$ mice. Toxicology, 209, 15-28. 2005.

[101] C.A. Granville et al. A central role for Foxp $3^{+}$regulatory T cells in K-Ras-driven lung tumorigenesis. PLoS One, 4, e5061. 2009.

[102] M. Khatami. 'Yin and Yang' in inflammation: duality in innate immune cell function and tumorigenesis. Expert Opin. Biol. Ther., 8, 1461-1472. 2008.

[103] J.M. Evans et al. Metformin and reduced risk of cancer in diabetic patients. BMJ, 330, 1304-1305. 2005.

[104] G. He et al. Thiazolidinediones inhibit insulin-like growth factor-i-induced activation of p70S6 kinase and suppress insulin-like growth factor-I tumor-promoting activity. Cancer Res., 66, 1873-1878. 2006. 
[105] G. Taubes. Cancer research. Cancer prevention with a diabetes pill? Science, 335, 29. 2012.

[106] E.M. Moran. Epidemiological and clinical aspects of nonsteroidal anti-inflammatory drugs and cancer risks. J. Environ. Pathol. Toxicol. Oncol., 21, 193-201. 2002.

[107] K. Wakabayashi. NSAIDs as cancer preventive agents. Asian Pac.J. Cancer Prev., 1, 97-113. 2000.

[108] N. C. Gorin E. Estey R. J. Jones H. I. Levitsky I., Borrello \& S. Slavin. New developments in the therapy of acute myelocytic leukemia. ASH Education Program Book, 2000(1), 69-89. 2000.

[109] B. Mayer. et al. Mitochondrial regulation of apoptosis. News Physiol. Sci., 18, 89-94. 2003.

[110] J.M. Adams. Ways of dying: multiple pathways to apoptosis. Genes Dev., 17, 2481-2495. 2003.

[111] M. R. Chinoy, M. M. Graybill, S. A. Miller, C. M. Lang \& G. L. Kauffman. Angiopoietin-1 and VEGF in vascular development and angiogenesis in hypoplastic lungs. American Journal of Physiology-Lung Cellular and Molecular Physiology, 283(1), L60-L66. 2002.

[112] H. Shim et al. c-Myc transactivation of LDH-A: implications for tumor metabolism and growth. Proc. Natl Acad. Sci. USA, 94, 6658-6663. 1997.

[113] V.R. Fantin et al. Attenuation of LDH-A expression uncovers a link between glycolysis, mitochondrial physiology, and tumor maintenance. Cancer Cell, 9, 425-434. 2006.

[114] C.M. Metallo et al. Reductive glutamine metabolism by IDH1 mediates lipogenesis under hypoxia. Nature, 481, 380-384. 2012.

[115] L. Dang, et al. Cancer-associated IDH1 mutations produce 2-hydroxyglutarate. Nature, 462, 739-744. 2009.

[116] J. Adam et al. Rare insights into cancer biology. Oncogene. 2013.

[117] L. Hue et al. Fructose 2, 6-bisphosphate and the control of glycolysis by growth factors, tumor promoters and oncogenes. Adv. Enzyme Regul., 33, 97-110. 1993.

[118] A. Yalcin et al. Regulation of glucose metabolism by 6-phosphofructo- 2-kinase/fructose-2, 6-bisphosphatases in cancer. Exp. Mol. Pathol., 86, 174-179. 2009.

[119] N. Méndez - Sánchez, M. Arrese, D. Zamora - Valdés \& M. Uribe, Current concepts in the pathogenesis of nonalcoholic fatty liver disease. Liver International, 27(4), 423-433. 2007.

[120] E. Rysman K. Brusselmans K. Scheys L. Timmermans R. Derua S. Munck \& F. Vanderhoydonc. De novo lipogenesis protects cancer cells from free radicals and chemotherapeutics by promoting membrane lipid saturation. Cancer research, 70(20), 8117-8126. 2010.

[121] M. S. Cooke M.D. Evans M. Dizdaroglu \& J. Lunec. Oxidative DNA damage: mechanisms, mutation, and disease. The FASEB Journal, 17(10), 1195-1214. 2003.

[122] Murphy, G. et al. Progress in matrix metalloproteinase research. Mol. Aspects Med., 29, 290-308. 2008.

[123] V. Serra et al. Extracellular superoxide dismutase is a major antioxidant in human fibroblasts and slows telomere shortening. J. Biol. Chem., 278, 6824-6830. 2003.

[124] P. Shelton et al. The transcription factor NF-E2-related factor 2 (Nrf2): a proto-oncogene? FASEB J., 27, 414-423. 2013.

[125] H. C. Chen P. C. Chan M. J. Tang C. H. Cheng \& T. J. Chang Tyrosine phosphorylation of focal adhesion kinase stimulated by hepatocyte growth factor leads to mitogen-activated protein kinase activation. Journal of Biological Chemistry, 273(40), 25777-25782. 1998.

[126] M.A. Le Bitoux et al. Tumor-host interactions: the role of inflammation. Histochem. Cell Biol., 130, 1079-1090. 2008.

[127] M.V. Maffini et al. Stromal regulation of neoplastic development: age-dependent normalization of neoplastic mammary cells by mammary stroma. Am. J. Pathol., 167, 1405-1410. 2005

[128] J. Yang, \& R.A. Weinberg, Epithelial-mesenchymal transition: at thess crossroads of development and tumor metastasis. Developmental Cell, 14(6), 818-829. 2008.

[129] D. S., Micalizzi, S. M., Farabaugh, \& H. L. Ford. Epithelial-mesenchymal transition in cancer: parallels between normal development and tumor progression. Journal of mammary gland biology and neoplasia, 15(2), 117-134. 2010 . 